LOGES, V.; LEMOS, M.A.; RESENDE, L.V.; MENEZES, D.; CANDEIA, J.A.; SANTOS, V.F. Resistência de cultivares e híbridos de cebola a tripes. Horticultura Brasileira, Brasília, v.22, n.2, p.222-225, abril-junho 2004.

\title{
Resistência de cultivares e híbridos de cebola a tripes
}

\author{
Vivian Loges'; Margarida A. Lemos ${ }^{1}$; Luciane V. Resende'; Dimas Menezes ${ }^{1}$; Jonas A. Candeia²; Venézio \\ F. dos Santos ${ }^{2}$ \\ ${ }^{1}$ UFRPE, Av. D. Manoel de Medeiros, s/n, 52171-900 Recife-PE; E-mail: vloges@yahoo.com; ${ }^{2}$ IPA, Av. Gal. San Martim, 1371, 50761- \\ 000 Recife-PE; E-mail: ipa@ipa.br
}

\section{RESUMO}

Foi avaliada a resistência a Thrips tabaci das cultivares de cebola ValeOuro IPA-11, Roxa IPA-3, Belém IPA-9, Texas Grano-502, Brownsville e Duquesa, e dos híbridos Dessex e Granex Ouro. O experimento foi conduzido em condições de campo em Juazeiro (BA) sem a utilização de controle químico. Caracteres de folha foram avaliados aos 26; 48 e 69 dias após o transplante (DAT) e caracteres de produção aos 93 DAT. O delineamento experimental foi em blocos ao acaso, com três repetições. A cv. Duquesa apresentou menor número de tripes associado ao menor número de folhas e maior ângulo entre as folhas centrais, sugerindo resistência por não-preferência. A cultivar Duquesa e os híbridos Dessex e Granex Ouro foram moderadamente resistentes para os caracteres de produção e para o caráter número de ninfas do inseto.

Palavras-chave: Allium cepa, Thrips tabaci, arquitetura da planta, produção, resistência, insetos, melhoramento, não preferência.

\begin{abstract}
Thrips resistance in onion cultivars and hybrids

Resistance to Thrips tabaci was estimated for onion cultivars ValeOuro IPA-11, Roxa IPA-3, Belém IPA-9, Texas Grano-502, Brownsville and Duquesa, and for the hybrids Dessex and Granex Ouro. The experiment was carried out under field conditions in Juazeiro, Bahia State, Brazil, without the use of chemical control. Leaf characters were evaluated at 26;48 and 69 days after transplanting date (DAT), and yield characters were evaluated at 93 DAT. The experiment design was total randomized blocks with three replications. The cv. Duquesa showed the lowest number of immature forms of T. tabaci, which was associated with the lowest number of leaves and the largest degree of insertion between the central leaves, suggesting a nonpreference type of resistance. The cv. Duquesa and hybrids Granex Ouro and Dessex were mode $\backslash$ rately resistant to the thrips based on yield characters and on the number of immature forms of the insect.
\end{abstract}

Keywords: Allium cepa, Thrips tabaci, plant architecture, yield, insects, resistance, breeding, nonpreference.

\section{(Recebido para publicação em 16 de julho de 2003 e aceito em 27 de fevereiro de 2004)}

$\mathrm{D}$ entre os fatores que causam baixos rendimentos na cultura da cebola no Brasil estão as infestações de Thrips tabaci, chegando a causar prejuízos de 45\% (Sato, 1989). Causam danos diretos que ocorrem pela sucção da seiva nos tecidos mais jovens, provocando diminuição da área foliar e decréscimo da capacidade fotossintética, reduzindo a produtividade, e danos indiretos por meio da transmissão de agentes fitopatogênicos (Candeia et al., 1998).

O método de controle mais utilizado é o químico, o que promove desequilíbrio biológico, aumento de riscos de contaminação de homens, animais e ambiente, favorece o surgimento de populações resistentes e aumenta os custos de produção. Controles alternativos como a utilização de cultivares de cebola menos suscetíveis ao ataque de tripes é uma das formas mais viáveis de reduzir seus prejuízos, podendo contribuir para o manejo integrado do tripes em cebola. Candeia et al. (1998) observaram que a cv. ValeOuro IPA-11 apresentou folhagem mais resistente aos danos por tripes, porém o tipo e causas da resistência não foram bem esclarecidos. A resistência de cebola a tripes está relacionada com a arquitetura da planta, isto é, ao formato e inserção das folhas (Sato, 1989; Jones et al., 1935). A avaliação deste caractere (arquitetura da planta), associado à produção, é de suma importância para a identificação de materiais resistentes a este inseto. O objetivo deste trabalho foi avaliar a resistência de cultivares e híbridos de cebola a T. tabaci.

\section{MATERIAL E MÉTODOS}

O experimento foi conduzido em Juazeiro (BA), na região do Submédio São Francisco. O transplantio das mudas foi realizado 37 dias após a semeadura, no espaçamento de $0,20 \mathrm{~m}$ x 0,15 $\mathrm{m}$, com ausência de seleção para tamanho de plantas. $\mathrm{O}$ delineamento experimental foi em blocos ao acaso, com oito tratamentos: as cultivares ValeOuro IPA-11, Roxa IPA-3, Belém IPA-9,
Texas Grano-502, Brownsville e Duquesa, e os híbridos comerciais Dessex e Granex Ouro, em três repetições. A irrigação foi feita por sulcos de infiltração e os demais tratos culturais foram os adotados na região, porém sem o uso de agroquímicos para controle de pragas após o transplantio.

A parcela experimental foi constituída por um leirão com 2,50 m de comprimento e 1,00 m entre sulcos de irrigação, sendo efetuadas três avaliações, aos 26; 48 e 69 dias após o transplantio (DAT), dos caracteres: comprimento de folhas (CF), correspondendo ao comprimento da bainha até o ápice da maior folha; ângulo entre as duas folhas centrais completamente desenvolvidas (ANG), tomado com o auxílio de um transferidor a partir do eixo central da planta e expresso em graus; número total de folhas (NTF); número de folhas danificadas por tripes (NFD), sendo consideradas as folhas com sinais de prateamento e amarelecimento; número de ninfas nas duas folhas centrais 
Tabela 1. Comprimento de folhas, ângulo entre as duas folhas centrais, total de folhas, folhas danificadas por tripes e ninfas nas duas folhas centrais em cultivares e híbridos de cebola. Juazeiro (BA), UFRPE, 1998.

\begin{tabular}{lrrrrrrrrrr}
\hline \multirow{2}{*}{ Tratamento } & \multicolumn{4}{c}{ 48 dias após o transplantio } & \multicolumn{4}{c}{ 69 dias após o transplantio } \\
\cline { 2 - 12 } & $\mathbf{C F}^{1}$ & ANG & NTF & NFD & NINFA & CF & ANG & NTF & NFD & NINFA \\
\hline ValeOuro IPA-11 & $42,55 \mathrm{a}$ & $9,46 \mathrm{a}$ & $7,53 \mathrm{ab}^{2}$ & $1,80 \mathrm{a}$ & $11,67 \mathrm{a}$ & $43,30 \mathrm{ab}$ & $6,47 \mathrm{bc}$ & $9,93 \mathrm{ab}$ & $9,73 \mathrm{a}$ & $27,00 \mathrm{ab}$ \\
Roxa IPA-3 & $42,24 \mathrm{a}$ & $5,67 \mathrm{a}$ & $6,80 \mathrm{~b}$ & $1,73 \mathrm{a}$ & $5,47 \mathrm{a}$ & $49,67 \mathrm{a}$ & $3,93 \mathrm{~d}$ & $10,20 \mathrm{a}$ & $9,47 \mathrm{a}$ & $41,93 \mathrm{a}$ \\
Belém IPA-9 & $40,65 \mathrm{a}$ & $6,33 \mathrm{a}$ & $7,73 \mathrm{ab}$ & $2,80 \mathrm{a}$ & $10,27 \mathrm{a}$ & $45,36 \mathrm{ab}$ & $5,53 \mathrm{c}$ & $9,87 \mathrm{ab}$ & $8,87 \mathrm{ab}$ & $44,00 \mathrm{a}$ \\
Texas Grano & $41,31 \mathrm{a}$ & $10,26 \mathrm{a}$ & $6,53 \mathrm{~b}$ & $2,33 \mathrm{a}$ & $7,13 \mathrm{a}$ & $39,63 \mathrm{~b}$ & $6,53 \mathrm{bc}$ & $8,60 \mathrm{abc}$ & $8,27 \mathrm{abc}$ & $16,33 \mathrm{bc}$ \\
Brownsville & $36,44 \mathrm{a}$ & $8,66 \mathrm{a}$ & $6,87 \mathrm{~b}$ & $2,27 \mathrm{a}$ & $7,80 \mathrm{a}$ & $39,03 \mathrm{~b}$ & $6,73 \mathrm{bc}$ & $8,13 \mathrm{bcd}$ & $7,53 \mathrm{abc}$ & $23,40 \mathrm{abc}$ \\
Duquesa & $46,51 \mathrm{a}$ & $5,67 \mathrm{a}$ & $7,67 \mathrm{ab}$ & $2,20 \mathrm{a}$ & $12,67 \mathrm{a}$ & $44,63 \mathrm{ab}$ & $9,40 \mathrm{a}$ & $6,13 \mathrm{e}$ & $6,13 \mathrm{c}$ & $1,27 \mathrm{~d}$ \\
Granex Ouro & $44,82 \mathrm{a}$ & $6,47 \mathrm{a}$ & $8,33 \mathrm{a}$ & $3,13 \mathrm{a}$ & $6,93 \mathrm{a}$ & $38,43 \mathrm{~b}$ & $8,00 \mathrm{ab}$ & $6,67 \mathrm{de}$ & $6,67 \mathrm{bc}$ & $8,07 \mathrm{~cd}$ \\
Dessex & $41,13 \mathrm{a}$ & $6,53 \mathrm{a}$ & $7,53 \mathrm{ab}$ & $2,40 \mathrm{a}$ & $15,60 \mathrm{a}$ & $37,77 \mathrm{~b}$ & $5,93 \mathrm{c}$ & $7,00 \mathrm{cde}$ & $6,93 \mathrm{bc}$ & $9,73 \mathrm{bcd}$ \\
\hline
\end{tabular}

${ }^{1} \mathrm{CF}$ - comprimento de folhas; ANG - ângulo entre as duas folhas centrais completamente desenvolvidas; NTF - número total de folhas; NFD - número de folhas danificadas por tripes; NINFA - número de ninfas nas duas folhas centrais completamente desenvolvidas.

${ }^{2}$ médias na coluna seguidas pela mesma letra não diferem entre si pelo Teste de Duncan $\mathrm{P}<0,05$.

completamente desenvolvidas (NINFA). A contagem dos insetos foi realizada segundo metodologia de Loges et al. (2000). As médias dos tratamentos foram comparadas por meio do teste de Duncan.

Para avaliação dos caracteres associados à produção, a parcela experimental foi constituída por um leirão com 8,5 $\mathrm{m}$ de comprimento. Quando $50 \%$ das plantas apresentaram o tombamento da folhagem, aos 93 DAT, todas as plantas da parcela foram colhidas, curadas por dois dias e realizada a toalete, eliminando-se as raízes e folhas. Os caracteres avaliados foram: produção de bulbos não comerciais (PBNC) em kg/parcela, sendo considerados os bulbos com diâmetro inferior a $40 \mathrm{~mm}$; produção de bulbos comerciais (PBC) em kg/parcela, correspondente ao peso de bulbos com diâmetro maior ou igual a $40 \mathrm{~mm}$; peso médio de bulbos comerciais (PMBC) em kg, relativo ao peso total de bulbos comerciais dividido pelo número de bulbos comerciais; porcentagem de plantas improdutivas (\%I), representadas por plantas com ausência de embulbamento (charutos e cebolões) em relação ao número de plantas sobreviventes na colheita; porcentagem de bulbos não comerciais (\%BNC) correspondendo à relação de bulbos não comerciais com o número de plantas sobreviventes na colheita; porcentagem de bulbos comerciais $(\% \mathrm{BC})$, calculado através da relação de bulbos comerciais com o número de plantas sobreviventes na colheita. Os tratamentos fo- ram classificados quanto ao grau de resistência a tripes com base no intervalo de confiança (IC) ao nível de 95\% para os caracteres de produção. Para PBC, PMBC e \%BC foram classificados em: moderadamente resistentes (MR) quando a média foi maior que o IC; suscetível (S) para média dentro do IC; altamente suscetível (AS) quando a média foi menor que o IC. Para os caracteres PBNC, \%I, \%BNC e NINFA, os tratamentos foram classificados de forma inversa (adaptado de Barros, 1998).

\section{RESULTADOS E DISCUSSÃO}

As amostras de tripes enviadas para identificação indicaram a ocorrência apenas da espécie $T$. tabaci L. (Monteiro, 2001). A infestação por $T$. tabaci na cultura da cebola, 26 dias após o transplantio (DAT), foi muito baixa, sendo desconsiderada. Para os caracteres de folha (Tabela 1), foram detectadas diferenças significativas $(\mathrm{P}<0,05)$ entre os tratamentos aos 48 DAT apenas para o número total de folhas (NTF), e aos 69 DAT para todos os caracteres avaliados.

Aos 69 DAT, a cv. Roxa IPA-3 apresentou maior comprimento de folha (CF) que as cvs. Texas Grano e Brownsville e híbridos Dessex e Granex Ouro. A cv. Belém IPA-9 apresentou altura das plantas inferior à observada por Duarte et al. (1998), no final do ciclo da cultura em Picos (PI). A cv. Duquesa apresentou, aos $69 \mathrm{DAT}$, o maior ângulo entre as folhas, enquanto a cv.
Roxa IPA-3, o menor ângulo. Houve redução do ângulo formado entre as folhas centrais completamente desenvolvidas dos 48 e aos 69 DAT em todos os tratamentos, com exceção da cv. Duquesa e do híbrido Granex Ouro, nos quais o ângulo aumentou, coincidindo com a redução da infestação por tripes.

O híbrido Granex Ouro apresentou o maior número de folhas aos 48 DAP enquanto as cvs. Roxa IPA-3, Texas Grano e Browsville o menor. Estes materiais não mantiveram estes resultados na avaliação aos 69 DAP, sendo o maior número de folhas observado na cv. Roxa IPA-3 e o menor na cv. Duquesa. Notase que entre as duas avaliações, em um intervalo de 21 dias, houve aumento no número total de folhas que variou de 1,2 a 3,4 folhas, com exceção da cv. Duquesa e dos híbridos Dessex e Granex Ouro. Dowker et al. (1976) observaram, em um intervalo de 28 dias (128 e 156 dias após o semeio), a formação de até 1,2 folhas em progênies de cebola.

$\mathrm{O}$ número de folhas danificadas (NFD) aos 48 DAP foi bem menor que o NTF em todos os genótipos. Já aos 69 DAP foi muito próximo ou igual ao NTF, indicando que quase todas as folhas apresentaram danos, reflexo do aumento da infestação de T. tabaci entre as duas avaliações. Segundo Ramiro (1972), a incidência do T. tabaci ocorre de forma desuniforme inicialmente, crescendo gradualmente, com maior população no final do ciclo da cultura. Este fato não foi observado na cv. Duquesa e no híbrido Dessex, observando- 
Tabela 2. Classificação quanto ao grau de resistência a tripes, para caracteres de produção, em cultivares e híbridos de cebola. Juazeiro (BA), UFRPE, 1998.

\begin{tabular}{lcccccccc}
\hline \multirow{2}{*}{ Tratamento } & \multicolumn{9}{c}{ 93 dias após o transplantio } \\
\cline { 2 - 9 } & PBNC & PBC & PMBC & \% I & \% BNC & \%BC & $\begin{array}{c}\text { NINFA 48 } \\
\text { DAT }\end{array}$ & $\begin{array}{c}\text { NINFA 69 } \\
\text { DAT }\end{array}$ \\
\hline ValeOuro IPA-11 & $0,69 \mathrm{~S}^{2}$ & $6,09 \mathrm{AS}$ & $72,20 \mathrm{AS}$ & $2,53 \mathrm{~S}$ & $25,90 \mathrm{AS}$ & $71,57 \mathrm{AS}$ & $11,67 \mathrm{AS}$ & $27,00 \mathrm{MR}$ \\
Roxa IPA-3 & $0,84 \mathrm{~S}$ & $6,41 \mathrm{AS}$ & $65,80 \mathrm{AS}$ & $8,58 \mathrm{AS}$ & $25,94 \mathrm{AS}$ & $65,49 \mathrm{AS}$ & $5,47 \mathrm{MR}$ & $41,93 \mathrm{AS}$ \\
Belém IPA-9 & $1,25 \mathrm{AS}$ & $5,55 \mathrm{AS}$ & $61,40 \mathrm{AS}$ & $5,12 \mathrm{AS}$ & $35,05 \mathrm{AS}$ & $59,83 \mathrm{AS}$ & $10,27 \mathrm{AS}$ & $44,00 \mathrm{AS}$ \\
Texas Grano & $0,81 \mathrm{~S}$ & $8,03 \mathrm{AS}$ & $78,90 \mathrm{~S}$ & $5,15 \mathrm{AS}$ & $27,06 \mathrm{AS}$ & $67,79 \mathrm{AS}$ & $7,13 \mathrm{~S}$ & $16,33 \mathrm{MR}$ \\
Brownsville & $1,09 \mathrm{AS}$ & $4,64 \mathrm{AS}$ & $59,60 \mathrm{AS}$ & $10,58 \mathrm{AS}$ & $35,26 \mathrm{AS}$ & $54,16 \mathrm{AS}$ & $7,80 \mathrm{~S}$ & $23,40 \mathrm{MR}$ \\
Duquesa & $0,25 \mathrm{MR}$ & $14,31 \mathrm{MR}$ & $108,90 \mathrm{MR}$ & $0,00 \mathrm{MR}$ & $9,31 \mathrm{MR}$ & $90,69 \mathrm{MR}$ & $12,67 \mathrm{AS}$ & $1,27 \mathrm{MR}$ \\
Granex Ouro & $0,47 \mathrm{MR}$ & $10,03 \mathrm{MR}$ & $82,00 \mathrm{MR}$ & $0,70 \mathrm{MR}$ & $14,37 \mathrm{MR}$ & $84,93 \mathrm{MR}$ & $6,93 \mathrm{~S}$ & $8,07 \mathrm{MR}$ \\
Dessex & $0,39 \mathrm{MR}$ & $9,90 \mathrm{MR}$ & $82,00 \mathrm{MR}$ & $0,21 \mathrm{MR}$ & $12,85 \mathrm{MR}$ & $86,94 \mathrm{MR}$ & $15,60 \mathrm{AS}$ & $9,73 \mathrm{MR}$ \\
\hline
\end{tabular}

${ }^{1}$ PBNC - produção de bulbos não comerciais kg/parcela; PBC - produção de bulbos comerciais kg/parcela; PMBC - peso médio de bulbos comerciais g; \% I - médias originais da porcentagem de plantas improdutivas ; \%BNC - médias originais da porcentagem de bulbos não comerciais; \%BC - médias originais da porcentagem de bulbos comerciais; NINFA - médias originais do número de ninfas nas duas folhas centrais completamente desenvolvidas; DAT - dias após o transplantio.

${ }^{2}$ Classificação quanto ao grau de resistência: MR - moderadamente resistente; S - suscetível; AS - altamente suscetível.

se redução na população do inseto provavelmente devido a modificações na arquitetura das plantas entre os 48 e 69 DAT. Segundo Kendall e Capinera (1987), a interação entre o T. tabaci e a cultura da cebola torna-se complexa devido às alterações na palatabilidade $\mathrm{e}$ concentrações nutricionais das folhas nos três estádios de desenvolvimento da cultura (pré-bulbo, bulbo e amadurecimento).

A cv. Duquesa apresentou, aos 69 DAT, a menor infestação de tripes, o menor número de folhas e o maior ângulo entre as folhas (Figura 1). O menor número de folhas pode ter proporcionado maior ângulo entre as folhas centrais, reduzindo a área de proteção para o inseto, acarretando a não-preferência pelas plantas e com isso diminuindo a infestação. Jones et al. (1935) observaram estas características de resistência na cv. White Persian, Coudriet et al. (1979) na cv. de cebola branca Nebuka e Bortoli et al. (1995) em genótipos de alho.

A cv. Roxa IPA-3, com comportamento inverso à cv. Duquesa, apresentou a maior infestação associada com o maior número de folhas e menor ângulo (Figura 1). Cultivares de cebola com folhas achatadas e com ponto de crescimento compacto promovem um melhor abrigo para os tripes, protegendo-os contra inimigos naturais e da ação dos inseticidas, e minimizando as condições

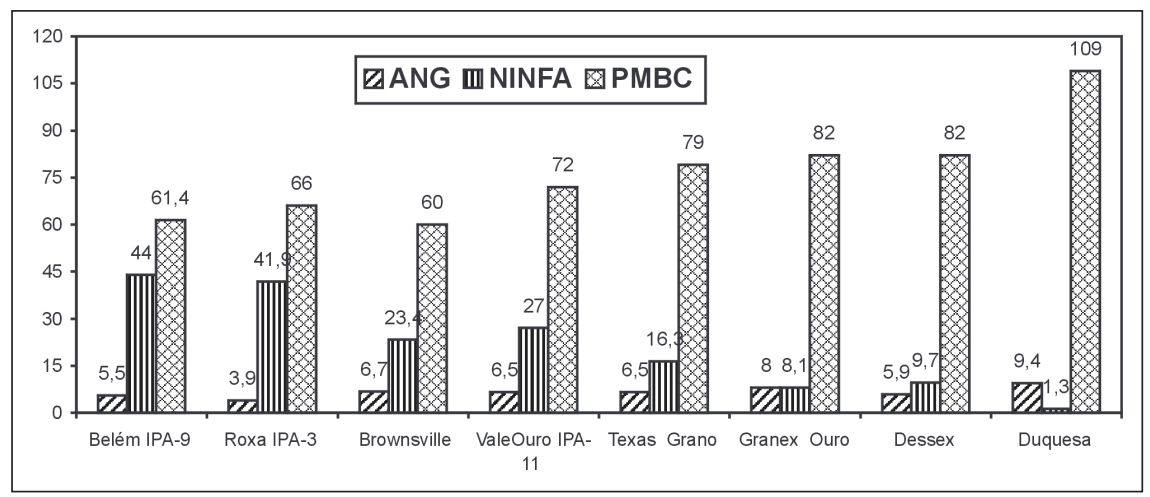

Figura 1. Ângulo (ANG) e ninfas de Thrips tabaci entre as duas folhas centrais (NINFA), 69 dias após o transplantio, e peso médio de bulbos comerciais (PMBC), em cultivares e híbridos de cebola. Juazeiro (BA), UFRPE, 1998.

adversas do ambiente (Jones et al., 1935). Plantas de alho com maior massa verde foram mais favoráveis ao desenvolvimento e aumento da infestação de tripes (Bortoli et al., 1995). Já Kendall e Capinera (1987) verificaram a correlação da produção de folhas adicionais no estágio de pré-bulbo com a capacidade de suportar os danos causados por tripes.

A cv. Duquesa e os híbridos Dessex e Granex Ouro foram classificadas como moderadamente resistentes (MR) para os caracteres associados à produção $\mathrm{e}$ NINFA aos 69 DAT (Tabela 2). Isto reforça que o tipo de resistência apresentado por estes genótipos possa ser a nãopreferência, uma vez que apresentaram infestação inferior a dez ninfas nas duas folhas centrais completamente desen- volvidas e os melhores resultados em relação à produção (Figura 1). Embora, aos 69 DAT, as cvs. ValeOuro IPA-11, Texas Grano e Brownsville tenham sido classificadas como MR para NINFA, foram suscetíveis (S) ou altamente suscetíveis (AS) considerando os demais caracteres associados à produção. Candeia et al. (1998) observaram que a cv. ValeOuro IPA-11 apresentou folhagem mais resistente aos danos por tripes.

As cvs. Roxa IPA-3 e Belém IPA-9 foram $\mathrm{S}$ ou AS com base nos caracteres associados à produção e NINFA aos 69 DAT, indicando que estas cultivares, em comparação com as outras, não apresentaram resistência por não-preferência. Candeia et al. (1998) afirmaram que a cv. Roxa IPA-3 apresentou tolerância a tripes. Hamilton et al. (1999) observa- 
ram que esta cultivar apresentou resistência por não-preferência devido, principalmente à característica estrutural da cera das folhas, e resistência por tolerância, uma vez que a produção em parcelas com controle químico não diferiu das parcelas sem controle químico.

Transformando os resultados de produção de bulbos comerciais (PBC) obtidos em metros quadrados para hectares, destaca-se a cv. Duquesa com produtividade de 16,83 t/ha, demonstrando que mesmo sem a aplicação de defensivos, ultrapassou a média nacional de $15,79 \mathrm{t} / \mathrm{ha}$ (IBGE, 2001), seguida dos híbridos Dessex e Granex Ouro, com produtividade de 11,65 e $11,80 \mathrm{t} / \mathrm{ha}$, respectivamente.

A cv. ValeOuro IPA-11 apresentou produtividade de $7,16 \mathrm{t} / \mathrm{ha}$, valor bem inferior ao observado por Costa et al. (2000) para a mesma região (Petrolina) de 39,54 t/ha e ao registrado por Duarte et al. (1998) em Picos de 42,07 t/ha.

A cv. Brownsville apresentou a menor produtividade $(5,46 \mathrm{t} / \mathrm{ha})$, mesmo tendo apresentado uma infestação de tripes em torno da média. Este pode ser um indicativo da maior suscetibilidade desta cultivar ao ataque de tripes, uma vez que para a mesma região, Costa et al. (2000) obtiveram produtividade de 55,38 t/ha, em condições ideais de cultivo. A cv. Roxa IPA-3 apresentou produtividade de 7,54 t/ha, abaixo da observada por Resende et al. (1996) de 12 a 19,2 t/ha, porém o peso médio de bulbos $(65,8 \mathrm{~g})$ obtido neste experimento foi superior ao observado por aqueles autores.

Os resultados demonstram que a cv. Duquesa e os híbridos Dessex e Granex
Ouro apresentaram as menores infestações de tripes, menores número de folhas, ângulos maiores entre as duas folhas centrais completamente desenvolvidas e maiores produções, indicando que o tipo de resistência envolvido possa ser a não-preferência. Estes genótipos podem ser indicados para o MIP em cebola, além de servirem de referencial ou genitores em cruzamentos para trabalhos de seleção e melhoramento visando resistência de cebola a tripes.

\section{AGRADECIMENTOS}

Os autores agradecem à FACEPE, ao IPA, ao Eng. Agrônomo Jarbas Florentino de Carvalho, a todos da Fazenda Rodeador e demais pessoas que contribuíram para a execução do trabalho.

\section{LITERATURA CITADA}

BARROS, R. Efeito de cultivares de repolho Brassica oleracea L. var. capitata na biologia da traça das cruciferas, Plutella xylostella (L. 1758) e do parasitóide Trichogramma pretiosum Riley, 1879. Piracicaba: ESALQ, 1998. 99 p. (Tese doutorado).

BORTOLI, S.A.; CASTELlANE, P.D.; TERASAKA, F. Incidência de tripes (Thrips tabaci Lind., 1888) (Thysanoptera-Thripidae) em genótipos de alho (Allium sativum L.). Cientifica, Jaboticabal, v.23, n.2, p.355-366, 1995.

CANDEIA, J.A.; CARVALHO, J.F.; MARANHÃO, E.A.A.; CAVALCANTI, V.A.L.B.; RODRIGUES, V.J.L.B. Avaliação do nível de resistência de populações de cebola ao tripes e ao "sapeca". In: CONGRESSO BRASILEIRO DE OLERICULTURA, 38., 1998, Petrolina. Anais... Petrolina: SOB, 1998.

COSTA, N.D.; RESENDE, G.M.; DIAS, R.C.S. Avaliação de cultivares de cebola em PetrolinaPE. Horticultura Brasileira, Brasília, v.18, n.1, p.57-60, mar. 2000.
COUDRIET, D.L.; KISHABA, A.N.; McCREIGHT, J.D.; BOHN, G.W. Varietal resistance in onions to thrips. Journal of Economic Entomology, v.72, p.614-615, 1979.

DOWKER, B.D.; HARDWICK, R.C.; FENNELL, J.F.M.; ANDREWS, D.J. Genotypic and enviromental correlations between leaf growth and bulb size in onion. Annals of Applied Biology, v.82, p.341-348, 1976.

DUARTE, R.I.R.; VELOSO, M.E.; ATHAYDE SOBRINHO, C. Avaliação de cultivares de cebola (Allium cepa L.) na microrregião de Picos-PI. In: CONGRESSO BRASILEIRO DE OLERICULTURA, 38., 1998, Petrolina. Anais... Petrolina: SOB, 1998.

HAMILTON, B.K.; PIKE, L.M.; SPARKS, A.N.; BENDER, D.A.; JONES, R.W.; CANDEIA, J.; FRANÇA, G. Heritability of thrips resistance in 'IPA-3' onion cultivar in South Texas. Euphytica, v.109, p.117-122, 1999.

IBGE. INSTITUTO BRASILEIRO DE GEOGRAFIA E ESTATÍSTICA. Levantamento sistemático da produção agrícola. Disponível em $<\mathrm{http}$ : /www.ibge.gov.br>. Consultado em 15 jun. 2001. JONES, H.A.; BAILEY,S.F.; EMSWELLER, S.L. Field studies of Thrips tabaci Lind. with especial reference to resistance in onions. Journal of Economic Entomology, v.28, n.4, p.678-680, 1935. KENDALL, D.M.; CAPINERA, J.L. Susceptibility of onion growth stages to onion thrips (Thysanoptera, Thripidae) damage and mechanical defoliation. Environmental Entomology, v.16, n.4, p.859-863, 1987.

LOGES, V; LEMOS, M.A.; MENEZES, D.; BARROS, R. Efficiency of methods for sampling thrips on onion. In: INTERNATIONAL CONGRESS OF ENTOMOLOGY, 11., 2000, Foz do Iguaçu, Anais ... Foz do Iguaçu: SEB, 2000. p.268. MONTEIRO, R.C. Identificação das amostras de tripes. f-consoli@tamu.edu. Mensagem pessoal. 6 mar. 2001.

RAMIRO, Z.A. Comportamento de variedades e controle do Thrips tabaci, Lindeman, 1888, em culturas de cebola (Allium cepa L.). Piracicaba: ESALQ, 1972. 144 p. (Tese doutorado).

RESENDE, G.M.; GOULART, A.C.P.; SILVA, R.A. Características de produção de cultivares de cebola em cultivo de verão. Horticultura Brasileira, Brasília, v.14, n.2, p.151-154, 1996.

SATO, M.E. Avaliação do dano e controle do Thrips tabaci Lindeman, 1888 na cultura da cebola (Allium cepa L.). Piracicaba: ESALQ, 1989. 93 p. (Tese doutorado). 